\title{
Factores de competitividad en las empresas hoteleras de Tijuana, Baja California
}

\author{
Ario Refugio Estrada Gaxiola* \\ Jorge Carlos Morgan Medina \\ Onésimo Cuamea Velázquez \\ Facultad de Turismo y Mercadotecnia \\ Universidad Autónoma de Baja California
}

\section{Resumen}

En el presente artículo se analiza el nivel de competitividad de los hoteles de tres, cuatro y cinco estrellas, de la ciudad de Tijuana, Baja California, a través de la revisión de la capacidad competitiva de la hotelería tijuanense, utilizando como herramienta de investigación la técnica cuantitativa, a través del involucramiento de los diversos factores que participan en la competitividad del sector hotelero. Previo al trabajo de campo, donde se obtuvo información de fuentes primarias, se consultaron fuentes secundarias para la construcción del marco conceptual del caso, el cual fundamenta los antecedentes y métodos del estudio.

\section{Palabras clave}

Competitividad, hotelería, turismo.

*Correo electrónico: ario@uabc.edu.mx, jorgemorgan@uabc.edu.mx, onesimo@uabc.edu.mx 


\title{
Competitiveness factors in the hotel industry of Tijuana, Baja California
}

\author{
Ario Refugio Estrada Gaxiola* \\ Jorge Carlos Morgan Medina \\ Onésimo Cuamea Velázquez \\ Facultad de Turismo y Mercadotecnia \\ Universidad Autónoma de Baja California
}

\begin{abstract}
This article reviews the competitiveness level of three, four and five star hotels in the City of Tijuana, Baja California by applying a quantitative research method that considers the different factors that determine the sectors competitiveness. An initial step consisted of the research of secondary information sources to allow the construction of the conceptual framework for the case that was followed by fieldwork where information from primary sources was obtained.
\end{abstract}

\section{KeY WORDS}

Systemic, lodging, tourism

*E-mail: ario@uabc.edu.mx, jorgemorgan@uabc.edu.mx, onesimo@uabc.edu.mx 


\section{Introducción}

La industria del turismo es un pilar de la economía de los países, y el caso de México no es la excepción. En Baja California, de los principales rubros de la actividad turística (medios de transporte, establecimientos de alimentos y bebidas y servicios de hospedaje) el sector hotelero requiere especial atención en cuanto a su comportamiento respecto al grado de desarrollo de sus estrategias competitivas.

Este estudio, sobre el caso específico de la ciudad de Tijuana, se centra en determinar las áreas competitivas y la oportunidad de las empresas de hospedaje, dada su cercanía con una de las entidades más ricas del mundo: el estado de California en Estados Unidos, con el que comparten frontera Baja California y Tijuana.

Debido a la relevancia de Tijuana y a su papel en el desarrollo del turismo en Baja California (por medio de la industria hotelera), se requieren estudios serios y profundos para establecer y estimular sus niveles de competitividad.

Para efectos de la investigación, se realizó un censo de las empresas hoteleras de tres, cuatro y cinco estrellas en Tijuana, con el objetivo primordial de evaluar las capacidades administrativas, de innovación, desarrollo tecnológico y de las competencias profesionales de quienes trabajan en estos hoteles. Asimismo, se diseñaron instrumentos de técnica cuantitativa de medición, teniendo en cuenta diversos factores esenciales para el desempeño competitivo de las empresas.

\section{Marco conceptual}

De acuerdo con varios autores, el concepto de competitividad no solo es difuso y complejo, sino incluso ambiguo (Varisco, 2008; Otero, 2006; Lotero, Moreno y Valencia, 2005), lo que conlleva una dificultad en el análisis de este paradigma, pues no hay una claridad indiscutible en la teoría.

Porter, uno de los máximos exponentes del tema de competitividad, en su obra La ventaja competitiva de las naciones (1991) deja en claro que "no existe una definición de competitividad ni una teoría de la misma para explicarla" (cit. en Otero, 2006: 10), y reconoce que su significado puede ser diferente dependiendo tanto de los fines para los cuales se utiliza -sea para las empresas 0 las naciones-, como de la disciplina o enfoque desde el cual se aborde. 


\section{Un acercamiento al concepto de competitividad}

A pesar de lo expuesto en el apartado anterior, Porter se aventura a definir la competitividad como "la capacidad para sostener e incrementar la participación de los mercados internacionales, con una elevación paralela del nivel de vida de la población” (Porter, 1991, cit. en Varisco, 2008: 11). El modelo que propone Porter (1991) es un "diamante" constituido por cuatro elementos interrelacionados (condiciones de factores -insumos-, contexto para la estrategia y rivalidad empresarial, condiciones de demanda e industrias relacionadas y de apoyo) y dos factores exógenos: el azar y el gobierno. Rugman (1991), a su vez, presenta un modelo que se diferencia del primero por incluir al gobierno en un pentágono de interrelación entre sus partes.

El modelo de Porter es justificable en el sentido de que las políticas gubernamentales pueden influir a todos los elementos, pero no se discute la manera en la cual los cuatro factores pueden o no afectar al gobierno (figura 1).

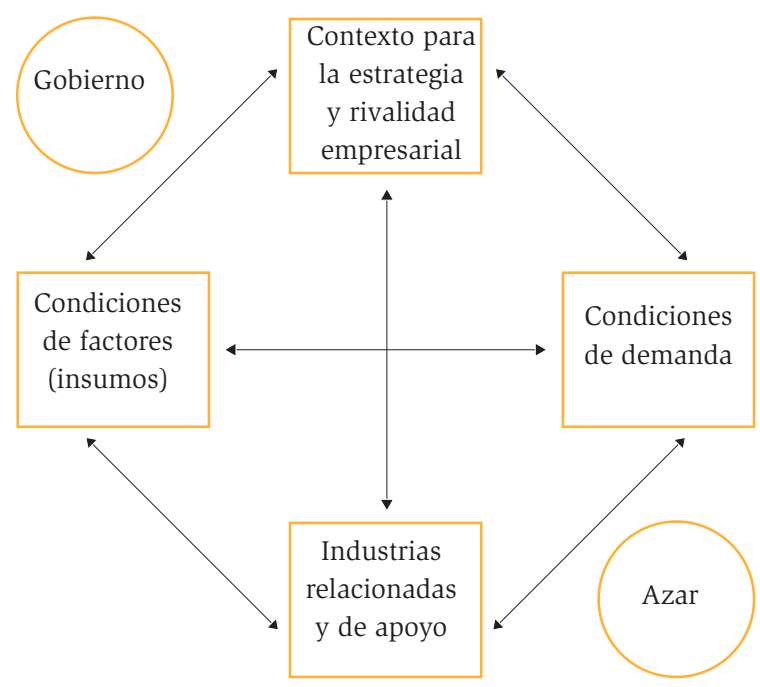

Fuente: Porter, M. (1991).

Figura 1. Diamante de la Competitividad de Porter 
Al no estar de acuerdo con la idea que Porter muestra en su diamante acerca del papel del gobierno, Rugman (1991) lo incluye como un quinto elemento determinante de la competitividad, y convierte el modelo en un "pentágono de la competitividad" (figura 2).

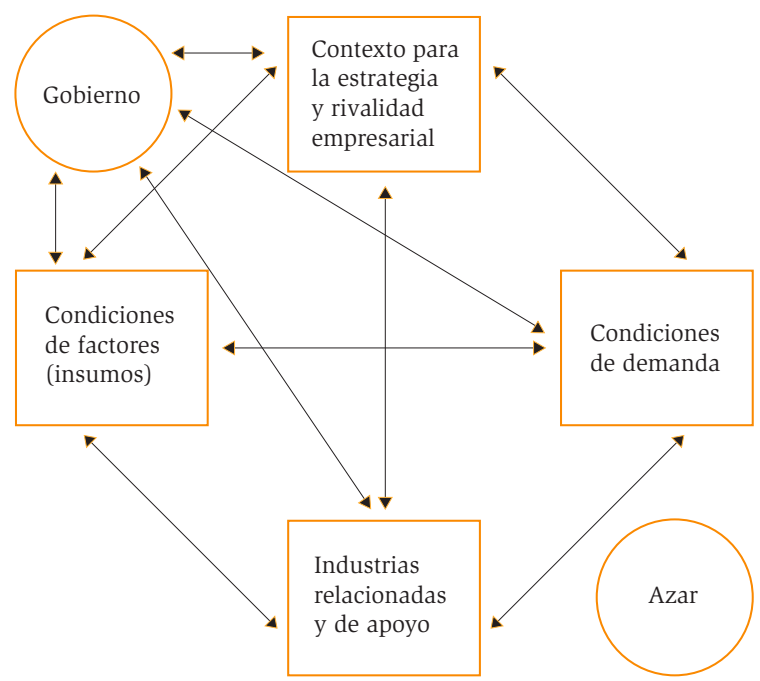

Fuente: Rugman, A. (1991).

Figura 2. Pentágono de la Competitividad de Rugman

Desde el enfoque del Institute for Management and Development (ImD), la competitividad en las empresas se debe, primordialmente, al ambiente competitivo que existe en las naciones; asimismo, las ventajas comparativas obedecen a su capital y las competitivas a la inclusión de tecnología como un factor de producción (IMD, 2007; cit. en Lombana y Rozas, 2009: 8).

En lo que respecta a las empresas, Fea (1995) define la competitividad como la capacidad estructural que les permite generar beneficios mediante los procesos de producción, de organización y de distribución; en ese sentido, la continuidad en la generación de beneficios es directamente proporcional al dinamismo estructural de la empresa; es decir, la competitividad se refiere a su capacidad para competir de manera exitosa y mantenerse en esa competencia. 
Otero (2006) explica la competitividad de las empresas en el sentido estricto de su desempeño comercial, y otorga esta misma característica a las industrias y a los países; para ello considera no solo el incremento de los activos de las empresas, sino también la mejora de la calidad de vida de la población, su bienestar social, además de la calidad de los empleos y ambiental, entre otros aspectos. De este modo, el incremento en el número de las empresas, así como su crecimiento, se debe a sus variados y complementarios instrumentos, por ejemplo: la integración vertical, la innovación, la gestión eficiente de los recursos, la incorporación de nueva tecnología, la descentralización de la producción, etcétera.

Otero (2006) relaciona tres niveles de competitividad: empresarial, nacional e industrial. La competitividad empresarial se asocia con la posibilidad de colocar sus productos de acuerdo con las características de las empresas y las de su entorno; la competitividad nacional se vincula con la capacidad de cierto país para generar un ambiente propicio para la competitividad industrial.

\section{La competitividad en dualidades}

En un sentido más amplio, es posible analizar y entender el concepto de competitividad en dualidades relacionadas. Así, se pueden establecer dualidades de análisis como las siguientes:

1. Competitividad espuria y competitividad genuina o auténtica. De acuerdo con Fajnzylber, la competitividad genuina o auténtica es aquella que representa una "mayor productividad, mayor eficiencia, mejor calidad, una diferenciación del producto, un sistema de distribución más adecuado” (1988: 7). Mientras que la espuria no se vale de instrumentos que conlleven un incremento del bienestar a largo plazo (particularidad de la competitividad genuina), sino que persigue el rápido aumento de la rentabilidad y de la participación en el mercado en el corto plazo.

2. Competitividad amplia y competitividad restringida. Según el IMD (2003, cit. en Otero, 2006: 14), la restringida se centra únicamente en el desempeño comercial de las firmas o el balance externo de una nación, y es definida 
como "el campo de la teoría económica que analiza los hechos y las políticas que le dan forma a la habilidad de una nación para crear y mantener un ambiente" para la competitividad. Por otro lado, la competitividad amplia debe mejorar la calidad de vida de la población y no solo la posición de la empresa, industria o nación en el comercio internacional.

3. Competitividad precio y competitividad tecnológica. La primera consiste en la producción de bienes o prestación de servicios con características iguales o similares a las de la competencia, pero a un mejor precio; es decir, se esfuerza por tener costos bajos para vender a precios bajos. Por su parte, la competitividad tecnológica se basa en la aplicación y el aprovechamiento de adelantos tecnológicos para elevar la productividad de las empresas y/o para ofrecer productos y servicios diferenciados (Otero, 2006).

4. Competitividad sistémica y competitividad no sistémica. Sánchez y Acosta (2001, cit. en Otero, 2006: 15) mencionan algunos enfoques de la competitividad no sistémica: $a$ ) el macroeconómico, que visualiza la competitividad como el resultado de las condiciones de variables "macro" y su influencia en los costos de producción y los precios de los factores; $b$ ) el neolaboralista, que afirma que la mano de obra calificada es primordial para asegurar una ventaja competitiva en los países, y que asigna un papel igual de relevante a la inversión y a la capacitación de la mano de obra; c) por último, el neoinstitucionalista, que otorga un rol esencial a los aspectos institucionales en materia de gestión, innovación y aprendizaje. Varios autores (Esser et al., 1996; Altenburg, Hillebrand y Meyer-Stamer, 1998) apuntan una visión sistémica, donde la competitividad no surge de forma espontánea al modificarse el contexto macro, ni se genera desde el espíritu de la empresa en el plano micro. En otras palabras, definen la competitividad sistémica como el resultado de un patrón de interacción compleja y dinámica entre el gobierno de un país, las empresas, las instituciones intermediarias y la capacidad organizativa de una sociedad; es decir, en cuatro niveles: micro, meso, macro y meta. 


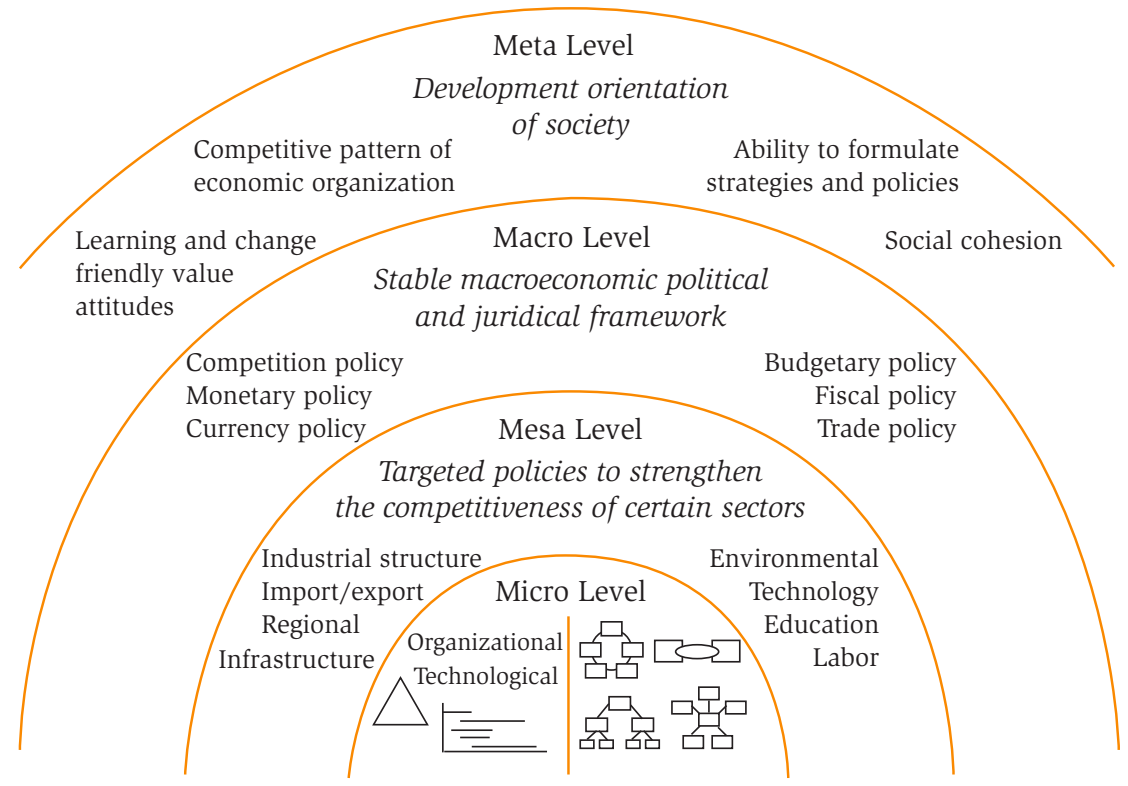

Fuente: Altenburg et al. (1998).

Figura 3. Competitividad Sistémica de Altenburg

5. Competitividad ex post y competitividad ex ante. Para Haguenauer (1989, cit. en Otero, 2006: 15), la primera se centra en el concepto de desempeño (ex post) y la segunda se vincula primordialmente con la productividad o eficiencia (ex ante). En el caso ex post, los productores pueden enfrentar a los competidores extranjeros en el mercado doméstico, mientras que en el ex ante el énfasis recae en la capacidad de producir ciertos bienes igualando o incluso superando los niveles de eficiencia de otras naciones.

6. Competitividad estática y competitividad dinámica. Pyke y Sengenberger (1992, cit. en Otero, 2006: 16) describen estos tipos de competitividad como la vía "baja” y la vía "alta”, respectivamente; la diferencia es que la competitividad estática se distingue por la competencia de precios, basándose en dotaciones factoriales, mientras que la dinámica se enfoca en la innovación. 


\section{El papel de las ventajas comparativa y competitiva en la competitividad}

A principios del siglo xix, partiendo de la teoría de la ventaja absoluta, David Ricardo desarrolla la teoría de la ventaja comparativa (Otero, 2006; Varisco, 2008); en ella predice que existen ganancias provenientes del comercio porque una nación produce en mayor cantidad el bien que le reporta una ventaja comparativa y en menor proporción otros bienes. De esta manera, un país puede exportar el bien que tiene una ventaja comparativa y así aumentar el consumo de ambos bienes. La ventaja comparativa está basada en varios supuestos (Otero, 2006), entre ellos se encuentran los siguientes:

a) Hay solo dos países, dos mercancías y un factor de producción (el trabajo)

b) Existe libre comercio

c) Hay perfecta movilidad de la mano de obra dentro de cada país, pero inmovilidad entre las dos naciones

d) Los costos de producción son constantes

e) No existen costos de transporte

f) El cambio tecnológico es inexistente.

De acuerdo con el modelo de Heckscher y Ohlin, los cambios en los precios (derivados del intercambio comercial) tienen fuertes efectos sobre las ganancias. "Dado que el comercio produce la convergencia de precios relativos, aumentando el valor de los bienes en los cuales el país posee ventajas comparativas y disminuyendo el precio de los otros, los propietarios de los factores abundantes en el país serán quienes ganen con el comercio, mientras que los propietarios de los factores escasos serán los perdedores” (Heckscher y Ohlin, s. f., cit. en Otero, 2006: 17).

En cuanto a la ventaja competitiva, Porter (1991) le da una gran relevancia a la innovación, y destaca el papel de la empresa como generadora de la competitividad nacional debido a que en ella se encuentra el proceso innovador. Para él, la principal característica de la ventaja competitiva es la innovación, la cual debe ser permanente, a pesar de que se requiera un esfuerzo continuo por mantenerla -porque la innovación puede ser imitada por los competidores-. También puede ser inusual, pues existen "fuerzas dentro de las organizaciones que desincentivan 
el cambio" (Porter, 1991, cit. en Otero, 2006: 19), como la presencia de un entorno desafiante, con presiones y adversidades para la empresa.

\section{La competitividad turística}

Entre las diferentes perspectivas desde las cuales se puede abordar el tema de la competitividad está la visión del turismo, sobre todo la de los propios destinos turísticos. En consecuencia, por competitividad del destino turístico puede entenderse "la capacidad del destino para crear e integrar productos con valor añadido que sostienen sus recursos, al tiempo que mantienen su posición en el mercado en relación a sus competidores" (Hassan, 2000: 240). Por otro lado, D’Hauteserre (2000: 21, 23 y 35) la define como "la capacidad de un destino para mantener su posición en el mercado y compartir y/o mejorarla a través del tiempo".

Varios autores han relacionado el término competitividad turística con la sostenibilidad, por ejemplo, Hassan (2000) señala que los destinos turísticos que establecen y aplican diversas estrategias orientadas hacia la sostenibilidad medioambiental del turismo consiguen tasas de crecimientos más elevadas. Para Ritchie y Crouch (2000), el destino turístico competitivo es aquel que promueve el máximo bienestar para sus habitantes de forma sostenible, el cual no solo debe ser económico, sino también ecológico, social, cultural y político. A su vez, Valls (2004: 56) afirma que "para que un destino turístico sea competitivo debe generar a largo plazo beneficios superiores a la media de la competencia en tres ámbitos": económico, social y medioambiental.

De acuerdo con Amaya, Conde y Covarrubias (2008: 17), "la ventaja competitiva en el turismo se refiere a la habilidad de un destino (turístico) para emplear de manera efectiva sus recursos en el largo plazo", también mencionan que las ventajas competitivas y comparativas son las bases para los modelos de competitividad turística, toda vez que esta se encuentra estrechamente ligada con el paisaje, la flora, la fauna, el clima y el patrimonio histórico y cultural (ventajas comparativas), y con la habilidad para emplear de modo efectivo sus recursos en el largo plazo (ventaja competitiva). 


\section{Antecedentes y contexto}

La Secretaría de Turismo del Estado de Baja California (Secture, 2002) indica que en 2002 se generaron 45807 empleos en el sector turístico (5.8 \% del total de empleos en la entidad), de los cuales 14604 (31.91 \%) se vinculaban con la industria manufacturera relacionada con el turismo (artesanías, recuerdos y curiosidades) y 771 (1.68 \%) dependían de la construcción de edificaciones turísticas. El rubro de comunicaciones y transportes produjo 3057 puestos de trabajo (6.67\%), y otros segmentos económicos: gastronómico, de eventos y de servicios, entre otros, con excepción del ramo hotelero, crearon 1128 empleos (aproximadamente $2.46 \%$ ).

Por su parte, la hotelería, los restaurantes y el comercio resultaron ser los de mayor peso para el turismo bajacaliforniano, pues de ellos dependían entonces 26247 empleos (57.3 \% de los trabajos asociados a la actividad turística) (figura 4).

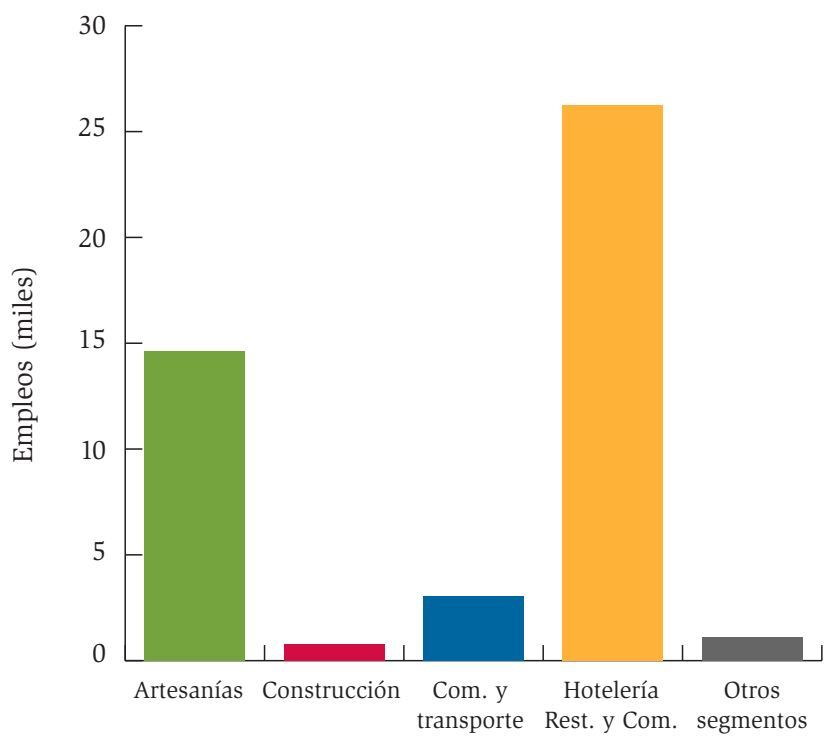

Fuente: Elaboración propia con base en Secture (2002). 
Es importante destacar que los datos obtenidos del estudio de Secture (2002: 26), único en su tipo, son proyectados y estimados "según la proporción del empleo en el turismo nacional con respecto al total de empleos generados en el país, esa misma proporción se aplica para el total de empleos generados en Baja California, y se obtiene en forma aproximada el número de trabajadores empleados en el sector turismo del Estado", por lo debe tenerse en cuenta lo endeble que puede resultar esta información, la cual, por consiguiente, debe ser tomada con mesura, sobre todo al utilizarla para la toma de decisiones.

Ahora bien, en cuanto a la ocupación hotelera en Baja California, de acuerdo con el Sistema Integral de Información de Mercados Turísticos (2014), en el año 2010 hubo 1883249 de cuartos ocupados, para 2011 la cifra creció a 1992 871, y para 2012 se reportaron 2066833 habitaciones ocupadas; lo que muestra un pequeño pero sostenido incremento en la ocupación de habitaciones en estos tres años (figura 5).

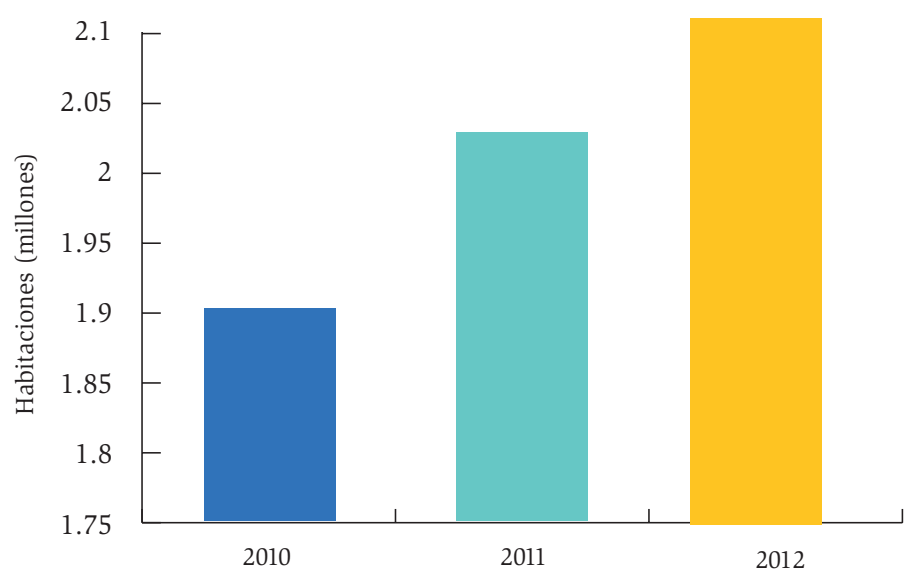

Fuente: Elaboración propia con base en Sistema Integral de Información de Mercados Turísticos (2014).

Figura 5. Total de habitaciones ocupadas en los años 2010, 2011 y 2012 en el estado de Baja California 
Se recomienda no solo dar su justa dimensión a la información presentada sino asimismo aprovechar para detectar áreas de oportunidad que atiendan vacíos de datos duros sobre el tema. La antigüedad de esta información revela la desatención a estos rubros y la consecuente disminución de la importancia del turismo como sector económico en el estado, pues a pesar de que a partir de 2010 su recuperación ha sido segura, también ha sido lenta.

\section{Planteamiento del problema}

Por lo descrito en el apartado anterior, se consideró pertinente realizar un estudio sobre los factores de competitividad del sector hotelero de la ciudad de Tijuana. Así pues, la investigación a partir de la cual se elaboró este artículo busca a) generar en el momento adecuado información objetiva y confiable sobre la competitividad de la hotelería en Tijuana y, ante la competencia mundial del sector, $b$ ) identificar las fortalezas, oportunidades y escenarios de desarrollo turístico de la ciudad, para que los resultados expuestos sean estimados como una fuente de información que brinde certeza y permita a los empresarios y al sector público contar con más y mejores herramientas para la toma de decisiones en beneficio de la actividad turística, desarrollar ventajas competitivas y optimizar sus relaciones de cooperación con los factores que intervienen en la prestación de servicios turísticos.

\section{Objetivo del estudio}

Analizar los diferentes factores de competitividad de las empresas hoteleras de tres, cuatro y cinco estrellas de la ciudad de Tijuana, Baja California.

\section{Descripción del método empleado}

El método utilizado para obtener los resultados de fuentes primarias fue de tipo cuantitativo, mediante la técnica de encuesta, para lo cual se aplicaron cuestionarios a gerentes y directivos responsables de la toma de decisiones en el sector hotelero. Una vez resueltos los cuestionarios, se procesaron los datos y, 
mediante el programa Statistical Package for the Social Sciences (SPss), versión 19.0, se consiguió la información estadística.

Para definir la población del estudio se tomó como referencia el directorio de hoteles y moteles de México y las estadísticas del Sistema Nacional de la Información Estadística del Sector Turismo de México (Datatur) (Colef-Secture, 2012), que establecen que en Baja California existen 63 hoteles y moteles de diferentes categorías; de esta base de datos se eligieron solo los de tres, cuatro y cinco estrellas ubicados en la ciudad de Tijuana que cumplieran con las características requeridas para el estudio; de un total de 18 hoteles únicamente participaron 12, los otros seis decidieron no colaborar en la investigación, decisión que fue respetada por el equipo. La relación de establecimientos de hospedaje de tres, cuatro y cinco estrellas que estuvieron involucrados en el trabajo de campo se presenta en el cuadro 1.

Cuadro 1. Relación de hoteles de tres, cuatro y cinco estrellas involucrados en el estudio

$\begin{array}{ll}\text { Hotel } & \text { CATEGORÍA } \\ \text { Grand Hotel Tijuana } & \star \star \star \star \star \\ \text { Marriot Tijuana } & \star \star \star \star \star \\ \text { Palacio Azteca } & \star \star \star \star \star \\ \text { Pueblo Amigo Inn } & \star \star \star \star \star \\ \text { Baja Inn Hacienda del Río } & \star \star \star \star \\ \text { Baja Inn La Mesa Inn } & \star \star \star \star \\ \text { Lausana Otay Aeropuerto } & \star \star \star \star \\ \text { Fiesta Inn Tijuana Otay } & \star \star \star \star \\ \text { Holiday Inn } & \star \star \star \star \\ \text { Ticuán Hotel } & \star \star \star \star \\ \text { España } & \star \star \star \\ \text { Aqua Río } & \star \star \star\end{array}$

Fuente: Trabajo de campo, 2013 


\section{Resultados del estudio}

Para identificar el nivel de calificación de cada puesto que el hotel contrata se utilizó una escala del 0 al 4; siendo 0 no calificado, 1 poco calificado, 2 medianamente calificado, 3 calificado y 4 altamente calificado.

Los encuestados consideran que deben estar calificados para el trabajo: el personal administrativo, de recepción, de amas de llaves, de ventas y reservaciones y el de seguridad; por otra parte, los empleados de alimentos y bebidas y de lavandería deben estar medianamente calificados (figura 6).

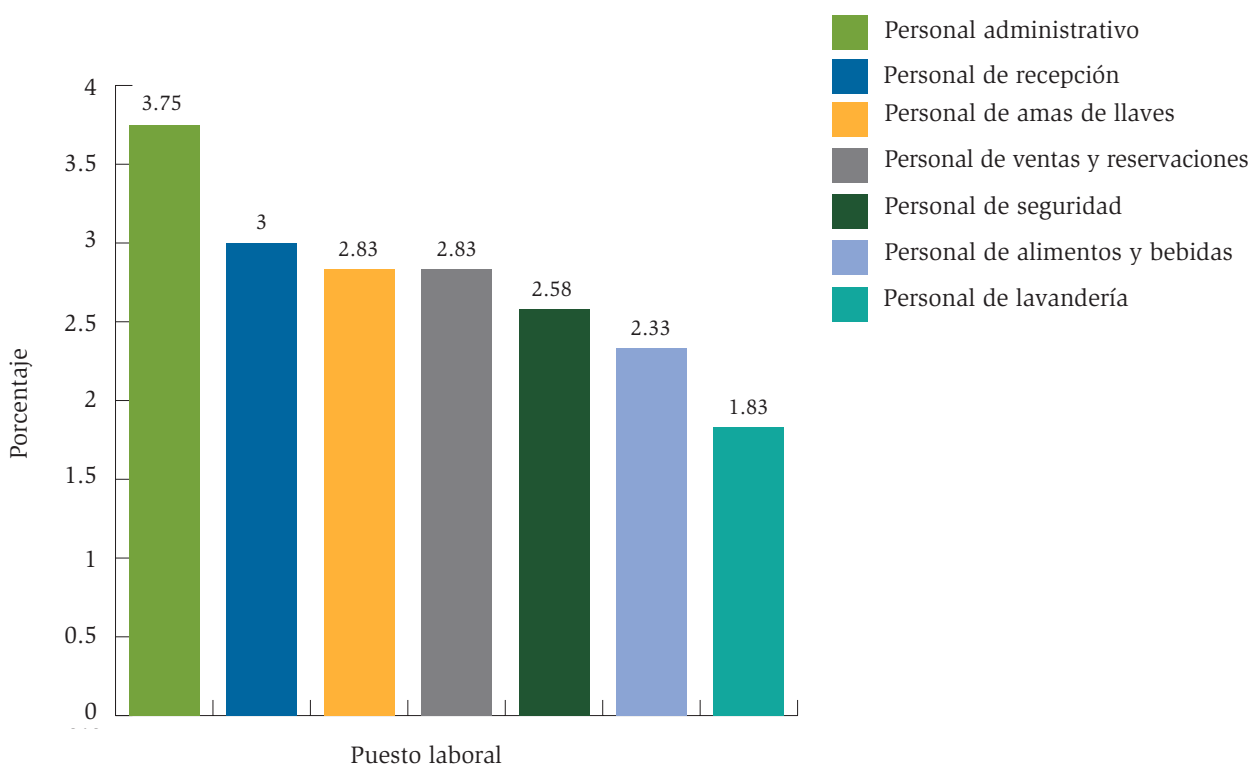

Fuente: Trabajo de campo, 2013.

Figura 6. Nivel de calificación requerido para diferentes puestos en los establecimientos de hospedaje de tres, cuatro y cinco estrellas en la ciudad de Tijuana, Baja California 
Para conocer la frecuencia con que se entrena y capacita a los empleados, así como el tipo de capacitación que se les da, se pidió a los encuestados que (de entre las respuestas que se describen en la gráfica 4), eligieran del 1 al 5 la frecuencia, siendo 5 mayor frecuencia, 4 muy frecuente, 3 frecuente, 2 poca frecuencia y 1 menor frecuencia (figura 7).

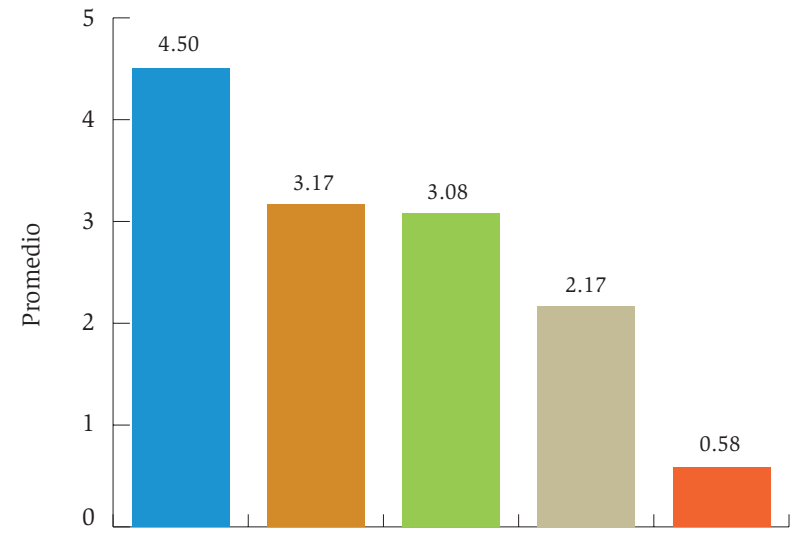

Al inicio de la contratación

Con un programa y presupuesto

Cuando se implementa un nuevo procedimiento de servicio

Basada en la Ley Federal del Trabajo

No los capacita

Manera de capacitación

Manera de capacitación

Fuente: Trabajo de campo, 2013.

Figura 7. Forma en la cual los hoteles de tres, cuatro y cinco estrellas entrenan y capacitan a sus empleados

En promedio, los participantes en el estudio reciben capacitación con mayor frecuencia al momento de ser contratados, con base en un programa y un presupuesto, y de manera frecuente cuando se pone en práctica un nuevo procedimiento o servicio. También se capacita a los empleados de acuerdo con lo establecido en la Ley Federal de Trabajo.

Por otro lado, se preguntó acerca del uso de sistemas que permitan medir la eficiencia de sus servicios, y se encontró que la mayoría (91.67 \%) sí lo hace; solo un hotel manifestó no emplear este recurso. 
En cuanto a los registros utilizados para detectar y llevar un control de los problemas que se suscitan al prestar los servicios, se encontró que $41.67 \%$ (cinco hoteles) emplea tanto estadísticas como bitácoras y reportes; $25 \%$ (tres establecimientos) solo bitácora. Otros hoteles llevan estadísticas, el control al día y licencias (LIC), entre otros tipos de registro (figura 8).

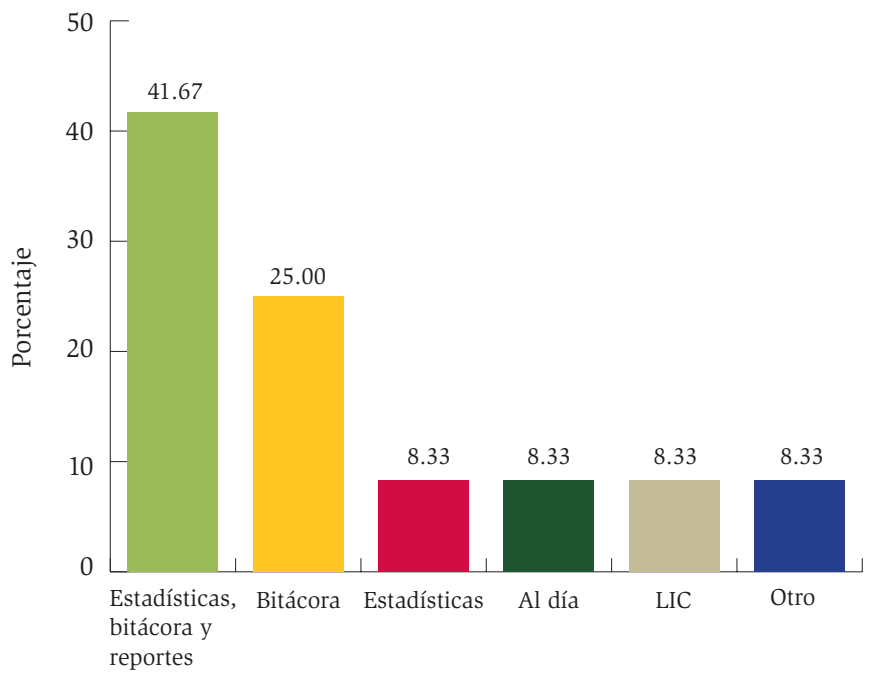

Tipo de registro

Fuente: Trabajo de campo, 2013.

Figura 8. Tipo de registro utilizado por hoteles de Tijuana para control de problemas presentados en el servicio

Ahora bien, acerca de las certificaciones, $25 \%$ de los entrevistados (tres hoteles) cuenta con Iso 9000; $16.67 \%$ (dos establecimientos) tiene la certificación AAA Five Diamonds, $16.67 \%$ el Distintivo "H" y el resto posee otros tipos de certificación. 
Cuadro 2. Certificaciones con las que cuentan los hoteles involucrados en el estudio

\begin{tabular}{|c|c|c|}
\hline \multicolumn{3}{|c|}{ CERTIFICACIONES CON LAS QUE CUENTAN LOS HOTELES } \\
\hline CERTIFICACIONES & Porcentaje & FRECUENCIA \\
\hline ISO 9000 & 25.00 & 3 \\
\hline AAA Five Diamonds & 16.67 & 2 \\
\hline Distintivo “ $\mathrm{H}$ ” & 16.67 & 2 \\
\hline Otras certificaciones & 41.67 & 5 \\
\hline & 100 & 12 \\
\hline
\end{tabular}

Fuente: Trabajo de campo, 2013

Sobre los mecanismos de evaluación del desempeño del personal, $53.85 \%$ recurre a la evaluación del jefe inmediato como el más común para medir la eficiencia de los trabajadores; 23.08 \% usa la evaluación del Departamento de Recursos Humanos. Otros establecimientos, 7.69 \% en cada caso, se sirven tanto de la evaluación del Departamento de Recursos Humanos como de la del jefe inmediato, la evaluación 360 y la apreciación del cliente.

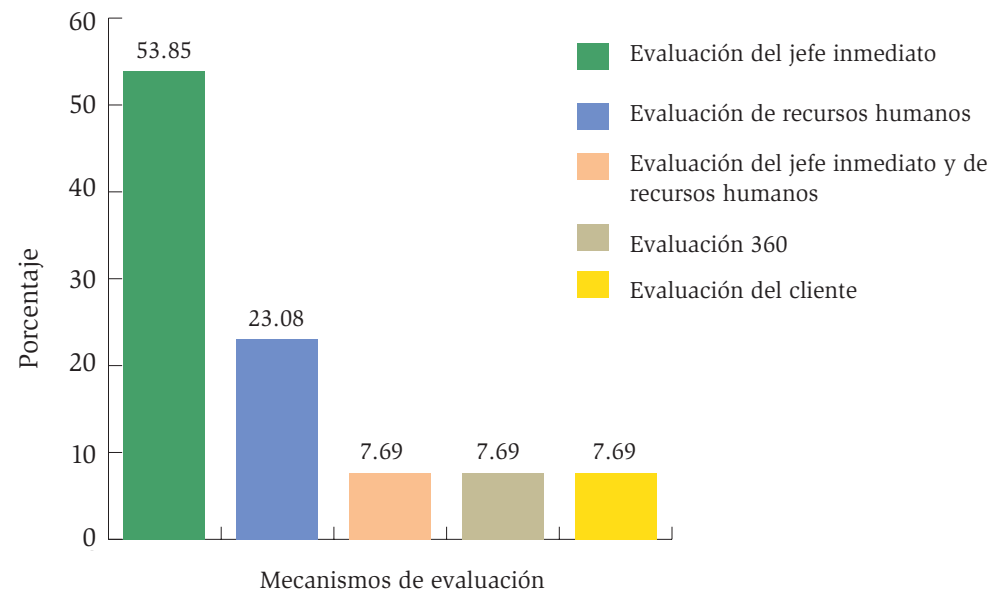

Fuente: Trabajo de campo, 2013.

Figura 9. Mecanismos de evaluación que utilizan los hoteles de tres, cuatro y cinco estrellas de Tijuana, para evaluar al personal en los 
Una tercera parte de los encuestados (cuatro hoteles) otorga bonos, propinas y comisiones dentro de sus programas de incentivos para sus empleados; $16.67 \%$ (dos hoteles) solo propinas; un establecimiento ( $8.33 \%$ de la muestra) tanto bonos como días de descanso, mientras que otro hotel da comisiones y reconocimientos al empleado del mes. Una cuarta parte de la muestra ( $25 \%$, conformado por tres hoteles) no contestó.

Para conocer en qué condiciones se encuentran las instalaciones generales del hotel, se pidió que seleccionaran una calificación del 0 al 4, siendo las condiciones: 4 excelentes, 3 buenas, 2 regulares, 1 malas y 0 pésimas. Para los participantes, las condiciones de las diferentes instalaciones (alcantarillado, red telefónica, red de agua potable y alumbrado) resultaron excelentes.

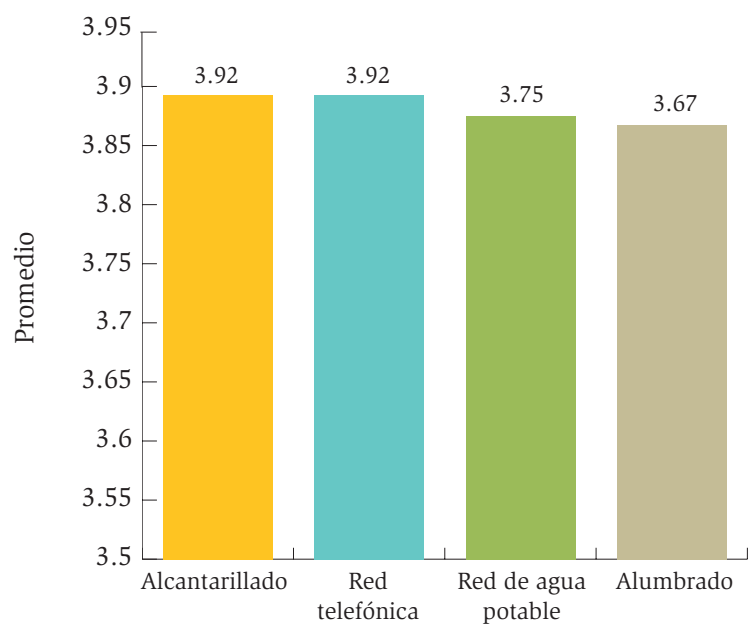

Instalaciones

Fuente: Trabajo de campo, 2013.

Figura 10. Condiciones de las instalaciones de los establecimientos

Acerca de las condiciones del equipamiento del hotel, también se solicitó que eligieran de la escala del 0 al 4 . Los encuestados evaluaron como excelentes las condiciones de las habitaciones y la recepción (con un promedio de 3.79); otros equipamientos, como estacionamiento, restaurante, cocina, bar, elevadores, áreas verdes y salones para eventos, fueron calificados con condiciones 
buenas (3.12 en promedio) y, por último, la alberca y el gimnasio fueron considerados regulares (con un promedio de 2.42).

Cuadro 3. Opinión de los hoteleros sobre las condiciones del equipamiento de sus establecimientos

\begin{tabular}{|l|c|}
\hline \multicolumn{2}{|c|}{ CondiCIONES DEL EQUIPAMIENTO DEL HOTEL } \\
\hline EquiPAMIENTO & PROMEDIO \\
\hline Habitaciones & 4 \\
\hline Recepción & 3.58 \\
Estacionamiento & 3.42 \\
Restaurante & 3.42 \\
Cocina & 3.33 \\
Bar & 3.25 \\
Elevadores & 2.83 \\
Áreas verdes & 2.83 \\
Salones para eventos & 2.75 \\
Alberca & 2.42 \\
Gimnasio & 2.42 \\
\hline
\end{tabular}

Fuente: Trabajo de campo, 2013

En cuanto a las condiciones de la tecnología del hotel (calificada con valores de 4 = excelentes, 3 = buenas, $2=$ regulares, $1=$ malas y $0=$ pésimas), los participantes dijeron que las condiciones de internet, hardware, sistemas y software son excelentes, con una puntuación promedio de 3.73. Los rubros con menor calificación (3.67) fueron los sistemas y el software; mientras que el mejor evaluado fue el internet (3.83). El hardware fue calificado en promedio con 3.75 . 


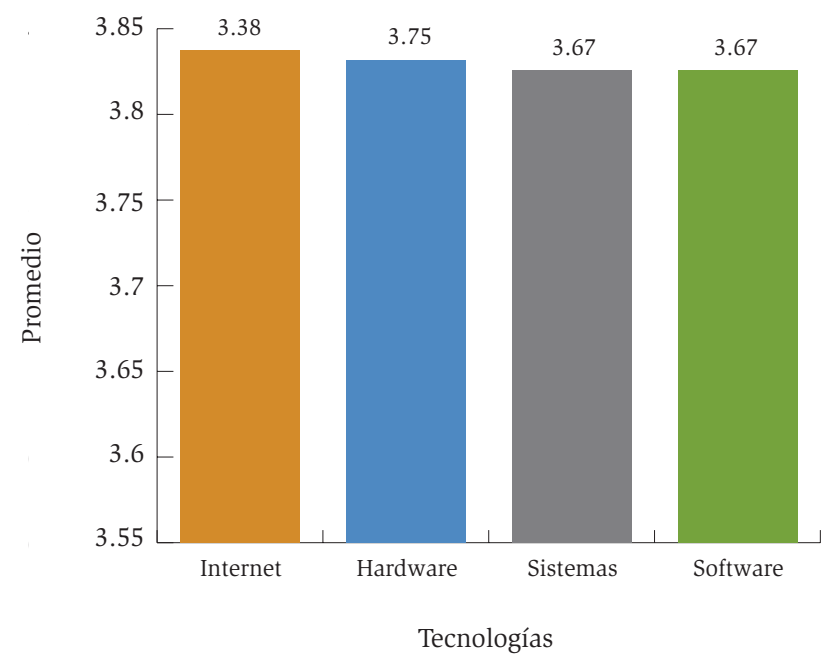

Fuente: Trabajo de campo, 2013.

Figura 11. Opinión de los hoteleros en relación a las condiciones en las que se encuentra la tecnología utilizada por el hotel

Se preguntó a los encuestados si sus establecimientos contaban con algún proyecto, programa o política de reducción de costos, y solo la mitad de ellos (seis) respondió que sí. Ahora bien, de los que si cuentan con un programa, proyecto o política de reducción de costos, $16.67 \%$ (un hotel) mencionó que sus programas se enfocan en reducir los consumos de agua, luz y papel. Otro hotel compara los precios de los insumos y servicios que utiliza, otro toma en cuenta la calidad del servicio que presta sin afectar su precio, y otro ha reducido el número de sus impresoras. El resto no contestó la pregunta (figura 12).

De quienes respondieron nuestra encuesta, $33.33 \%$ (cuatro menciones) opinó que la mayor ventaja competitiva con la que contaba su hotel era el servicio a los huéspedes; $16.67 \%$ (dos entrevistados) dijo que era la marca. El resto ( 8.33 \% en cada caso) indicó su ubicación, la publicidad, la calidad, la limpieza y la infraestructura. Solo un entrevistado no respondió a la pregunta (cuadro 4). 


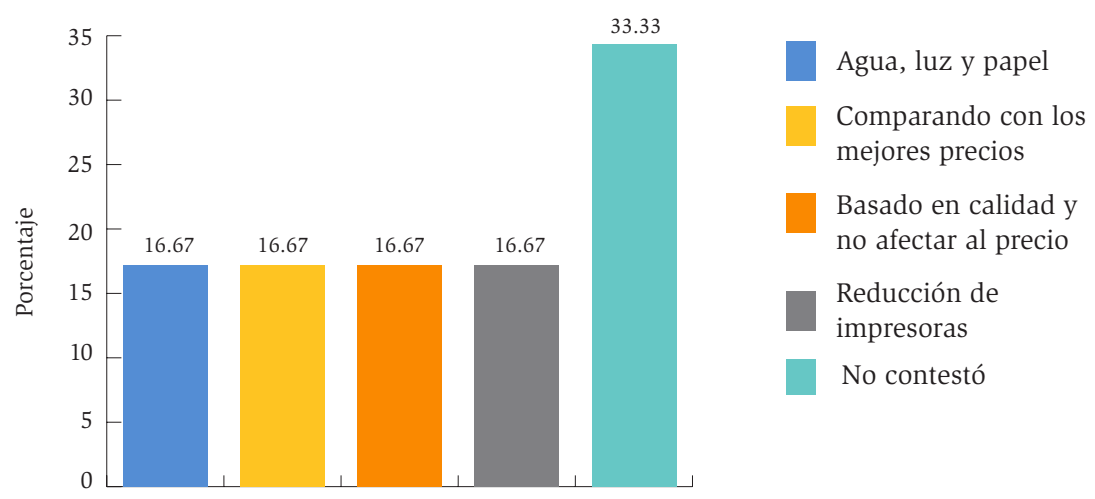

Función

Fuente: Trabajo de campo, 2013.

Figura 12. Finalidad o función que persiguen los proyectos, programas o políticas de las empresas para reducir costos.

Cuadro 4. Ventaja competitiva de mayor importancia de sus empresas, desde la perspectiva del hotelero de Tijuana

\begin{tabular}{|c|c|c|c|}
\hline \multicolumn{4}{|c|}{ VENTAJA COMPETITIVA DE MAYOR IMPORTANCIA PARA LA EMPRESA } \\
\hline VENTAJAS & & Porcentaje & FRECUENCIA \\
\hline Servicio & & 33.33 & 4 \\
\hline Marca & & 16.67 & 2 \\
\hline Ubicación & & 8.33 & 1 \\
\hline Publicidad & & 8.33 & 1 \\
\hline Calidad & & 8.33 & 1 \\
\hline Limpieza & & 8.33 & 1 \\
\hline Infraestructura & & 8.33 & 1 \\
\hline \multirow[t]{2}{*}{ No contestó } & & 8.33 & 1 \\
\hline & Total & 100 & 12 \\
\hline
\end{tabular}

Fuente: Trabajo de campo, 2013 
Para conocer con qué frecuencia realizan acciones de mejora continua, los encuestados eligieron del 1 al 3 la frecuencia con la que imparten capacitación al personal, siendo 3 muy frecuentemente (de tres a cuatro veces al año), 2 frecuentemente (de una a dos veces al año) y 1 ocasionalmente (que abarca un lapso de dos años). Las respuestas coincidieron en que la mejora de las instalaciones, el seguimiento del proceso de mejora continua, la capacitación del personal operativo, la mejora de equipamiento, la actualización del personal administrativo y la actualización de software y hardware se llevan a cabo frecuentemente; es decir, de una a dos veces al año.

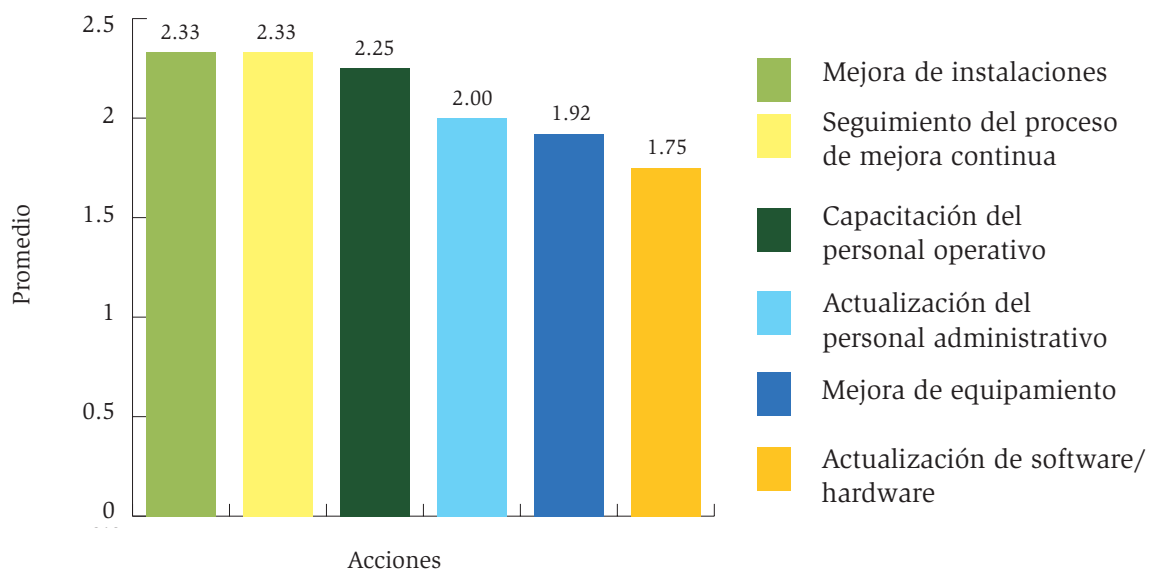

Fuente: Trabajo de campo, 2013.

Figura 13. Frecuencia con la que hoteles de Tijuana realizan acciones de mejora continua

Para conocer el valor de las tecnologías de la información y la comunicación (TIC) a partir de su uso en áreas administrativas, se solicitó se indicara del 1 al 5 su nivel de uso, tomando en cuenta que 5 representa el nivel más alto y 1 el más bajo. Los participantes concuerdan en que las áreas donde más se utilizan las Tic son contabilidad, finanzas, administrativa, informática, compras, recursos humanos y publicidad; y en menor grado en almacén, mercadotecnia, ventas, mantenimiento y relaciones públicas. 
Cuadro 5. Nivel de utilización de las tic en las diferentes áreas de los hoteles de Tijuana

\begin{tabular}{lc} 
ÁREAS & PROMEDIO \\
Contabilidad & 4.42 \\
Finanzas & 4.25 \\
Administrativa & 4.25 \\
Informática & 4.00 \\
Compras & 3.67 \\
Recursos Humanos & 3.58 \\
Publicidad & 3.50 \\
Almacén & 3.42 \\
Mercadotecnia & 3.17 \\
Ventas & 3.08 \\
Mantenimiento & 3.08 \\
Relaciones Públicas & Promedio general \\
\hline
\end{tabular}

Fuente: Trabajo de campo, 2013

Del total de los 12 representantes de los hoteles de cuatro y cinco estrellas de Tijuana, $\mathbf{6 6 . 6 7 \%}$ afirmaron que cada año se invierte en la mejora y actualización de sus instalaciones, equipamiento y tecnología. Una cuarta parte lo hace cada dos años, y 8.33 \% cada tres años o más.

Dos terceras partes de la muestra consideran que la situación financiera actual de su empresa es "buena”, mientras que el resto estima que es "regular".

\section{A manera de conclusión}

Con la finalidad de cumplir el objetivo del estudio sobre los diferentes factores de competitividad de los hoteles de cuatro y cinco estrellas de la ciudad de Tijuana, se llegó a las siguientes conclusiones: 
- Estos hoteles requieren un mayor nivel de calificación en sus empleados de acuerdo con el puesto que ocupan dentro de la organización.

- La manera en la que los hoteles capacitan y entrenan a sus empleados responde normalmente a necesidades ineludibles relacionadas con la contratación y el desempeño en sus puestos, y no se vincula a acciones de mejora continua o de calidad en el servicio.

- A pesar de que la mayoría de los hoteles aplican sistemas o programas de medición de la eficiencia en la prestación de sus servicios, sus acciones resultan muy subjetivas; no tienen programas que permitan detectar problemas en estos servicios y que conduzcan a una verdadera mejora continua de su calidad.

- Es necesario fomentar la incursión en diversos sistemas externos de certificación que conlleven un compromiso institucional; es decir, realizar más de una certificación (como las que se hacen hasta ahora), que complementen aquellas con que ya cuentan los establecimientos.

- Desde la perspectiva de los hoteleros, los establecimientos de hospedaje de Tijuana incurren en alguna forma de evaluación del desempeño del personal, pero estas son muy subjetivas y apegadas a las distintas obligaciones de los altos mandos, y no se detectan mecanismos o sistemas formales de evaluación, ni sistemas de estímulos e incentivos para el personal.

- En la opinión de los encuestados, las condiciones de sus instalaciones son óptimas.

- $\quad$ El equipamiento de los hoteles (albercas, elevadores, habitaciones, etc.) necesita ser atendido y mejorado de manera urgente; a pesar de que cuenta con un nivel aceptable, debe tener altos niveles de calidad para que los servicios que se prestan no se vean afectados.

- Las tecnologías utilizadas (software, hardware, servicios de internet y sistemas informáticos para la operación de los establecimientos) se encuentran en excelentes condiciones. 
- Así como se requiere la instrumentación de sistemas que conduzcan a la mejora continua de la calidad de los servicios y de la capacitación y entrenamiento de sus empleados, es necesario aplicar sistemas que reduzcan sus costos de producción, más allá de meros esfuerzos poco fructíferos nacidos de una supervisión endeble.

- De acuerdo con los hoteleros que participaron en la muestra, las principales ventajas competitivas de sus establecimientos son el servicio que prestan y su "marca".

- En los hoteles de cuatro y cinco estrellas de la localidad se realizan actividades de mejora continua en las instalaciones, la capacitación, el equipamiento, la actualización del personal administrativo y del software y hardware de una a dos veces al año.

- Los hoteles utilizan las Tic en todas sus áreas, pero estas son de gran relevancia en contabilidad, administración, finanzas e informática.

- Las empresas invierten en la actualización y mejora de sus instalaciones, equipamiento y tecnología cada uno o dos años aproximadamente.

- Dos terceras partes de los hoteles de cuatro y cinco estrellas de Tijuana tienen una buena situación financiera, pero el resto no.

Como conclusión general puede mencionarse que estos establecimientos tienen mucho por hacer para ser considerados competitivos. Esta labor, concerniente a sus altos mandos, no debe efectuarse de manera aislada ni limitada en el tiempo, sino que debe ser constante, integral e integrada, teniendo en cuenta las condiciones externas de las empresas.

\section{Fuentes consultadas}

Altenburg, T., W. Hillebrand y J. Meyer-Stamer (1998). Building Systemic Competitiveness. Concept and Case Studies from Mexico, Brazil, Paraguay, Korea and Thailand. Berlín: German Development Institute (Reports and Working Papers, 3). 
Amaya, C., E. Conde y R. Covarrubias (2008). "La competitividad turística: imperativo para Manzanillo, Colima”. Teoría y Praxis. Turismo, Negocios, Recursos Naturales, 5, 17-32.

Colef-Secture (2012). Inventario de establecimientos turísticos en Baja California. Disponible en: http://observaturbc.org /mapas /municipio/2\% 20Hoteles/1\%200ferta \% 20de\%20servicios \% 20turisticos/1\%20Unidades \%20de\%20hospedaje\%20por \%20categoria/web/StatPlanet.html

D'Hauteserre, A. (2000). "Lessons in Managed Destination Competitiveness:

The Case of Foxwoods Casino Resort”. Tourism Management, 21 (1), febrero, 23-32.

Esser, K. et al. (1996). "Competitividad sistémica. Nuevo desafío a las empresas y a la política”. Revista CEPAL, 59, agosto, 39-52.

Fajnzylber, F. (1988). "Competitividad internacional, evolución y lecciones". Revista de la CEPAL, 36, diciembre, 7-24.

Fea, Ugo (1995). Competitividad es calidad total. México: Alfaomega.

Hassan, S. (2000). "Determinants of Market Competitiveness in an Environmentally Sustainable Tourism Industry". Journal of Travel Research, 38, 239-245.

IMD (2007). IMD World Competitiveness Yearbook 2007: The Big Shake Up! Lausana: Institute for Management and Development.

Lombana, J. y S. Rozas (2009), "Marco analítico de la competitividad. Fundamentos para el estudio de la competitividad regional”, Pensamiento $y$ Gestión, 26, enero-junio, 1-38.

Lotero, J., A. Moreno y M. Valencia (2005). "La competitividad: aproximación conceptual desde la teoría del crecimiento y la geografía económica”. Borradores del CIE, 13, 1-25.

Otero, G. (2006). Competitividad: marco conceptual y análisis sectorial para la provincia de Buenos Aires. Buenos Aires: Gobierno de la Provincia de Buenos Aires (Cuaderno de Economía, 74).

Porter, M. (1991). La ventaja competitiva de las naciones. Buenos Aires: Vergara. ------ (2009). Estrategia competitiva: técnicas para el análisis de la empresa y sus competidores. Madrid: Pirámide.

Ritchie, J. y G. Crouch (2000). "The Competitive Destination. A Sustainability Perspective”. Tourism Management, 21, 1-7. 
Rugman, A. (1991). “Diamond in the Rough”. Business Quarterly, 55 (3), 61-64. Secture (2002). Impacto del sector turismo en la economía de Baja California [en línea]. Tijuana: Secretaría de Turismo del Estado de Baja CaliforniaGobierno del Estado de Baja California. Disponible en: http://www. descubrebajacalifornia.com/files/estudios/istebc.pdf $[2013,23$ de marzo].

Sistema Integral de Información de Mercados Turísticos (2014). Reporte de actividad hotelera por estado [en línea]. Disponible en: http://201.159.142.19/ Cognos/cgi-bin/cognos.cgi?b_action = cognosViewer\&ui. action $=$ run\&ui.object $=\% 2$ fcontent $\% 2$ fpackage $\% 5 b \% 40$ name $\% 3$ d $\% 27$ siimt_master $\% 27 \% 5 \mathrm{~d} \% 2$ freport $\% 5 \mathrm{~b} \%$ 40name $\% 3 \mathrm{~d} \% 27 \mathrm{ACTI}$ VIDAD_HOTELERA_ESTADO $\% 27 \%$ dd\&ui.name = ACTIVIDAD_HOTELERA_ESTADO\&run.outputFormat $=[2015,18$ de febrero $]$.

Valls, J. (2004). Gestión de destinos turísticos sostenibles. Barcelona: Ediciones Gestión 2000.

Varisco, C. (2008). "Desarrollo turístico y desarrollo local: La competitividad de los destinos turísticos de sol y playa”. Tesis de maestría en Ciencias Sociales. Buenos Aires: Universidad Nacional de Mar del Plata. 